hep-ph/0104074

MADPH-01-1219

\title{
Radion effects on unitarity in gauge-boson scattering
}

\author{
Tao Han, Graham D. Kribs, and Bob McElrath \\ Department of Physics, University of Wisconsin, \\ 1150 University Ave., Madison, WI 53706-1390 \\ than@pheno.physics.wisc.edu, kribs@pheno.physics.wisc.edu, \\ mcelrath@pheno.physics.wisc.edu
}

\begin{abstract}
The scalar field associated with fluctuations in the positions of the two branes, the "radion", plays an important role determining the cosmology and collider phenomenology of the Randall-Sundrum solution to the hierarchy problem. It is now well known that the radion mass is of order the weak scale, and that its couplings to standard model fields are order $\mathcal{O}\left(\mathrm{TeV}^{-1}\right)$ to the trace of the energy momentum tensor. We calculate longitudinal vector boson scattering amplitudes to explore the constraints on the radion mass and its coupling from perturbative unitarity. The scattering cross section can indeed become non-perturbative at energies prior to reaching the $\mathrm{TeV}$ brane cutoff scale, but only when some curvature-Higgs mixing on the $\mathrm{TeV}$ brane is present. We show that the coefficient of the curvature-Higgs mixing operator must be less than about 3 for the 4 -d effective theory to respect perturbative unitarity up to the $\mathrm{TeV}$ brane cutoff scale. Mass bounds on the Higgs boson and the radion are also discussed.
\end{abstract}




\section{Introduction}

Theories with extra dimensions have received tremendous attention within the last a few years. One of the most interesting incarnations was formulated by Randall and Sundrum (RS1) [1], who postulated a universe with two 4-d surfaces ("branes") bounding a slice of 5-d AdS spacetime. The Standard Model (SM) fields are assumed to be located on one brane (the "TeV brane"). Gravity lives on the other brane (the "Planck brane") and in the bulk as well as the TeV brane. Both branes have equal but opposite tension, while the bulk has a (negative) cosmological constant. By carefully tuning the brane tensions against the bulk cosmological constant, one can achieve a low energy effective theory that has flat 4-d spacetime. The RS1 metric takes the form

$$
d s^{2}=e^{-2 k L y} \eta_{\mu \nu} d x^{\mu} d x^{\nu}-L^{2} d y^{2}
$$

where $L$ is the size of the extra dimension and $0 \leq y \leq 1$. All mass scales in the full 5 - $\mathrm{d}$ theory are of order the Planck scale. By placing the SM fields at $y=1$, all mass terms must be rescaled by an exponential suppression factor ("warp factor") $e^{-k L}$ that can bring them down to the $\mathrm{TeV}$ scale. This merely requires that $L \sim 35 / k$, and thus roughly 35 times the fundamental Planck length. This is a dramatic improvement over the original hierarchy problem between the electroweak scale and the 4-d Planck scale $M_{\mathrm{Pl}}$.

The first obvious difficulty with this scenario is to arrange that the extra dimension stabilizes to a size of about an order of magnitude larger than the Planck length. In the original proposal, the potential for the size of the extra dimension is flat, so that all sizes are classically equivalent. The actual size of the extra dimension was tuned appropriately to solve the hierarchy problem. A more serious concern was first identified by Ref. [2], in which enforcing $d L(t) / d t=0$ in a cosmological context implied a nontrivial relationship between the $\mathrm{TeV}$ and Planck brane energy densities. Ultimately this was shown to be a direct result of assuming the potential for the size of the extra dimension is flat. Hence, the scalar field associated with fluctuations of the size of the extra dimension, the "radion", is massless. If, on the other hand, bulk dynamics setup a potential whose minimum determined the distance separating the two branes, then the radion acquires a mass.

Goldberger and Wise (GW) [3] noticed that a potential could be setup for the radion, by adding a 5-d bulk scalar to RS1 arranged so that it acquires an $y$-dependent vacuum expectation value (vev). Furthermore, the classical potential is stable against quantum corrections औ. In their analysis, the 5-d metric was generalized to

$$
d s^{2}=e^{-2 k L(x) y} \eta_{\mu \nu} d x^{\mu} d x^{\nu}-L(x)^{2} d y^{2}
$$

where $L$ has a vev and an $x_{\mu}$-dependent fluctuation. This generalization with radial fluctuations, however, does not satisfy Einstein's equations, and so a different ansatz for the metric is needed to

\footnotetext{
${ }^{1}$ In our conventions, the metric has signature $(+,-,-,-)$, Greek indices $\mu, \nu$, etc., run from $0,1,2,3$ denoting ordinary 4-d spacetime. The extra dimension is assumed to be compactified on a $S_{1} / Z_{2}$ orbifold.
} 
calculate the mass and couplings of the radion. Charmousis, Gregory, and Rubakov [5] proposed the metric

$$
d s^{2}=e^{-2 A(y)-2 F(x)} \eta_{\mu \nu} d x^{\mu} d x^{\nu}-(1+2 F(x)) d y^{2}
$$

in which they showed that a consistent treatment of the radial fluctuations characterized by the scalar field $F(x)$ is possible while solving the linearized Einstein equations. (In the RandallSundrum model, $A(y)=k L y$.)

This analysis, however, did not take into account the effect of the bulk scalar field back onto the metric (the "back-reaction"). Including the back-reaction of the bulk scalar field on the metric is in general highly nontrivial. However, DeWolfe, Freedman, Gubser, and Karch [6] proposed an interesting generating solution technique motivated from gauged supergravity. Their technique allows one to calculate the potential for the size of the extra dimension consistently including the effects of the bulk scalar field vev profile into the metric. This manifested itself through additional $y$-dependent terms in $A(y)$. However, radial fluctuations were not considered.

This motivated the work by Csáki, Graesser, and Kribs [7] (see also [8]) that used the generating solution technique by DeWolfe et al. [6], combined with the CGR metric ansatz [5], to show that a consistent treatment of the fluctuations in the size of the extra dimension could be done. In this work, the wave-function, mass, and couplings of the radion were explicitly calculated. In particular, it was shown that the mass of radion is of order $\mathrm{TeV} / 35$, multiplied by the size of the back-reaction (taken as a perturbation). Remarkably, this closely matched the radion mass calculation in Refs. [3, 9, 10], that used the "naive" ansatz in Eq. (2). The radion couplings could be obtained through

$$
r \frac{\delta S}{\delta r}=r \frac{\delta S}{\delta g_{\mu \nu}} \frac{\delta g_{\mu \nu}}{\delta r}=\frac{r}{e^{-k L} M_{\mathrm{Pl}}} T_{\mu}^{\mu}
$$

and thus couples to the trace of the energy momentum tensor $T_{\mu \nu}$. Here $r$ is the canonically normalized radion field, related to $F(x)$ via [7]

$$
F(x)=\frac{1}{\sqrt{6} M_{\mathrm{Pl}} e^{-k L}} r(x) .
$$

The strength of the radion couplings are proportional to the inverse "warped" Planck scale, and thus for appropriate choice of $L$, the $\mathrm{TeV}$ scale. One of the new results of this analysis is that the Kaluza-Klein (KK) excitations of the bulk scalar field couple to the SM fields, albeit suppressed by the size of the back-reaction divided by the KK mass (of order the warped Planck scale).

With a radion mass that is of order the weak scale, and couplings that are of order $1 / \mathrm{TeV}$, several groups proceeded to analyze the phenomenology of the radion [11, 12, 13]. It was quickly realized that the radion couplings are analogous to the Higgs at tree-level. (At one loop, the radion couples to the trace anomaly [11, 12], and therefore has a significantly different coupling to, for example, massless gauge bosons.) In particular, the tree-level couplings of the radion to the electroweak gauge bosons are the same as those of the SM Higgs, upon substituting

$$
h \longrightarrow-\gamma r, \text { with } \gamma=\frac{v}{\sqrt{6} \Lambda},
$$


where $h, r$ are the Higgs and radion mass eigenstates, $v=246 \mathrm{GeV}$ is the electroweak vev, and the warped Planck scale is $\Lambda=e^{-k L} M_{\mathrm{Pl}}$ [9, 10, 7]. Hereafter, we will use "TeV brane cutoff scale" or just "cutoff scale" to refer to the warped Planck scale, since our 4-d effectively theory is valid only up to about $\Lambda$. This leads to new contributions to the electroweak precision observables, such as the oblique corrections [7]. Generally, the size of this effect is rather small, since for a cutoff scale of order a TeV, $\gamma$ is order 0.1. For much lower cutoff scales, direct KK graviton production is important, and can provide constraints on the RS1 scenario [14].

Since the radion couplings to the SM are similar to those of the Higgs boson, it is natural to ask if the radion has any significant effects on the electroweak symmetry breaking sector, especially in the pessimistic scenario where the SM Higgs is rather heavy and may not be easily produced at collider experiments. In this paper we consider the effects of the radion on perturbative unitarity bounds in the SM. A few papers [15, 16] have considered some of the effects of the radion on unitarity involving external Higgs bosons, although no explicit description of the Goldstone boson equivalence theorem is present, nor the effects of including curvature-Higgs mixing. The paper is organized as follows. In Sec. 2 w wive a brief discussion of unitarity issues in the SM, and the bound on the Higgs mass that results. In Sec. 3 we introduce the 4-d effective theory that includes the radion and write the relevant interactions for gauge boson scattering. In Sec. 3.1 we calculate the partial wave amplitude including the effects of the radion to the largest process in the SM, namely $W^{+} W^{-} \rightarrow W^{+} W^{-}$, and show that there is in general no significant constraint on the radion mass or coupling in the absence of other interactions. In Sec. 3.2 we explicitly demonstrate that the Goldstone boson equivalence theorem can be applied, and thus in the high energy limit (large $s$ ) one obtains the same result replacing the longitudinally polarized $W$ 's by the eaten Goldstone bosons. The above analysis, however, neglected curvature-Higgs Higgs mixing (localized on the $\mathrm{TeV}$ brane). In Sec. 4 , we introduce the mixing, and recalculate the partial wave amplitude. We find that with a mixing coefficient $|\xi| \gtrsim 2.7$, the partial wave amplitude for $W$ scattering does exceed the unitarity bound for scattering energies lower than the cutoff scale. Finally, in Sec. 5 we present our conclusions.

\section{Perturbative unitarity}

In the $\mathrm{SM}$, the longitudinal components of the electroweak gauge bosons $\left(W_{L}^{ \pm}, Z_{L}\right)$ arise from the eaten Goldstone bosons resulting from the spontaneous breaking of the electroweak gauge symmetry. The study of scattering of longitudinally polarized gauge bosons would thus be the most direct means to explore the mechanism of the electroweak symmetry breaking. In this section, we briefly review the physics with longitudinal gauge boson scattering in the SM and discuss perturbative unitarity bounds. This serves as the basis for our further study including the radion.

We focus on the process

$$
W_{L}^{+} W_{L}^{-} \rightarrow W_{L}^{+} W_{L}^{-}
$$

since, in the high energy limit, this gives the largest contribution to the partial wave amplitude 


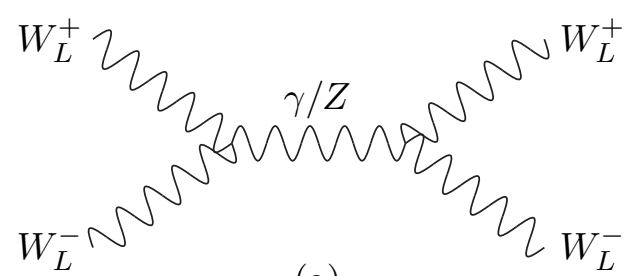

(a)

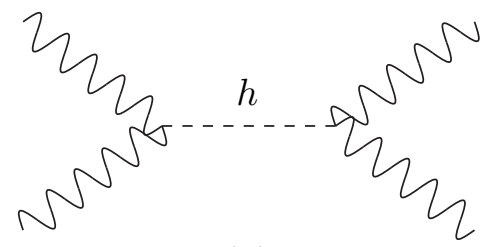

(d)

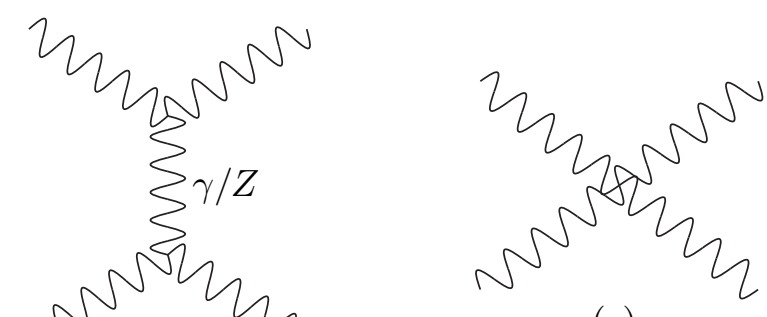

(b) (c)

Figure 1: Tree-level Feynman diagrams for $W W$ scattering in the SM.

of all $2 \rightarrow 2$ electroweak gauge boson scattering processes in the SM. The Feynman diagrams are shown in Fig. 11. At high energies, the wave-function of a longitudinal gauge boson can be written as

$$
\epsilon_{\mu}^{W_{L}}(p)=\frac{p_{\mu}}{M_{W}}+\mathcal{O}\left(\frac{M_{W}}{E}\right)
$$

This indicates that the longitudinally polarized gauge bosons are behaving as their eaten Goldstone boson counterparts with corrections of order $M_{W} / E$ where $E$ is the gauge boson energy.

With this substitution, the contributions from the diagrams in Fig. 1(a), (b), or (c) individually leads to a contribution $\mathcal{O}\left(s^{2} / M_{W}^{4}\right)$, but these terms cancel among each other due to electroweak gauge symmetry. The leading order result for the sum of these three diagrams, neglecting $\mathcal{O}\left(M_{W}^{2} / s\right)$ terms, is

$$
-i \mathcal{M}=-\frac{u}{v^{2}}
$$

At some point, the contribution to the partial wave amplitude is sufficiently large that the partial wave unitarity will be violated. This can be seen from the zeroth order partial wave amplitude

$$
a_{0}=\frac{1}{32 \pi} \int_{-1}^{1} d \cos \theta(-i \mathcal{M})=-\frac{s}{32 \pi v^{2}} .
$$

If we interpret the partial wave unitarity bound to be

$$
\left|\operatorname{Re}\left(a_{0}\right)\right|<\frac{1}{2}
$$

then one obtains the bound $\sqrt{s}<1.7 \mathrm{TeV}$. This is commonly referred as the indication of a strongly interacting electroweak sector at the TeV scale [17]. On the other hand, by including 
the Higgs contribution of Figs. 11(d) and (e), the amplitude becomes

$$
-i \mathcal{M}_{S M}=-\frac{m_{h}^{2}}{v^{2}}\left(\frac{s}{s-m_{h}^{2}}+\frac{t}{t-m_{h}^{2}}\right) \rightarrow-\frac{2 m_{h}^{2}}{v^{2}} \quad \text { for } \quad m_{h}^{2} \ll s,|t| .
$$

A light Higgs boson thus naturally restores partial wave unitarity, through a precise cancellation of the high energy divergence of the gauge boson scattering amplitude against the additional physical Higgs contribution. This mysterious cancellation becomes transparent by simply replacing the full massive spin-1 external gauge boson fields by the scalar Goldstone bosons, as dictated by the equivalence theorem [18, 17, 19]. However, this also implies an upper bound on the Higgs boson mass $m_{h}<900 \mathrm{GeV}$ if perturbative unitarity is maintained to arbitrarily high energies [18].

Although the unitarity bound cannot predict any specific form of new physics in the electroweak symmetry breaking sector, it does provide a general argument for a scale (around $1 \mathrm{TeV}$ ) at which the physics responsible for EWSB must show up. Furthermore, any new physics that couples to the EWSB sector significantly at high energies may be subject to constraints from partial wave unitarity.

\section{The radion effects}

In Randall-Sundrum scenarios, the low energy 4-d effective theory includes the ordinary 4-d graviton, the radion, and the KK excitations of the graviton and the bulk scalar. Here we are assuming the SM remains on the TeV brane (see e.g., Refs. [14, 20] for alternatives). At energies well below the cutoff scale, we need only consider the radion as the additional degree of freedom in our effective theory. As we approach (or exceed) the cutoff scale $\Lambda$, additional contributions from the KK modes (and perhaps other quantum gravity states) must be included in the calculations. However, including these contributions is subtle and model-dependent. For example, in the holographic viewpoint 21], the holographic dual 4-d theory is becoming strongly coupled in the transition to a 4-d conformal field theory above $\Lambda$. In any case, we will restrict ourselves to energies below the cutoff scale, and consider only the contribution from the radion.

As stated in the introduction, the radion couples to fields localized on the SM brane through

$$
-\frac{\gamma}{v} r T_{\mu}^{\mu}
$$

which leads to the three-point interaction terms

$$
\mathcal{L}=-\gamma \frac{2 M_{W}^{2}}{v} r W_{\mu}^{+} W^{-\mu}-\gamma \frac{M_{Z}^{2}}{v} r Z_{\mu} Z^{\mu}
$$

between the radion and the electroweak gauge bosons. The Lagrangian written above is not complete [7], since there are additional gauge-fixing terms that must be added. In addition, in dimensional regularization the Lagrangian is continued into $d$ dimensions, and there are yet more terms with coefficients that depend explicitly on $(d-4)$. Both of these sets of terms, however,

will not be relevant here since we are exclusively considering tree-level scattering amplitudes. 


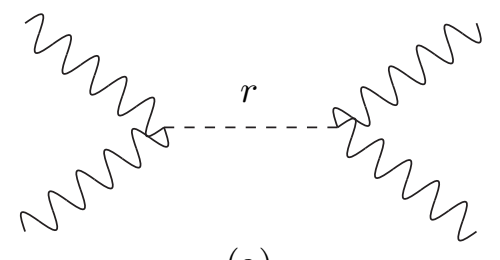

(a)

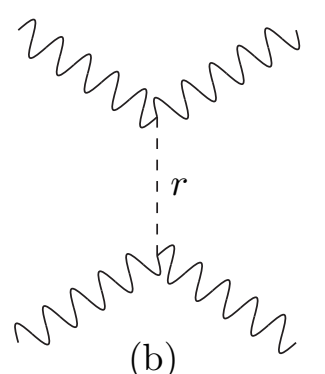

(b)

Figure 2: Tree-level Feynman diagrams for $W$ scattering through a radion.

We now proceed to calculate the scattering amplitude using gauge bosons, and then compare by doing the same calculation using Goldstone bosons.

\subsection{Radion contributions to $W_{L}^{+} W_{L}^{-}$scattering}

Since the radion couplings of Eq. (14) are analogous to the Higgs, there are additional contributions to electroweak gauge boson scattering from radion exchange. The radion contributes two additional diagrams to $W_{L}^{+} W_{L}^{-} \rightarrow W_{L}^{+} W_{L}^{-}$scattering, as shown in Fig. 2. The amplitude for the sum of the two contributions at high energy, neglecting $\mathcal{O}\left(M_{W}^{2} / s\right)$ terms as before, is

$$
\begin{aligned}
-i \mathcal{M}_{r}=-g^{2} \gamma^{2} & {\left[\frac{s}{4 M_{W}^{2}}+\frac{t}{4 M_{W}^{2}}+\frac{m_{r}^{2}}{2 M_{W}^{2}}+\frac{m_{r}^{2}}{4 M_{W}^{2}}\left(\frac{m_{r}^{2}}{s-m_{r}^{2}}+\frac{m_{r}^{2}}{t-m_{r}^{2}}\right)\right.} \\
& \left.+2 \frac{t}{s}+\left(\frac{M_{W}^{2}}{m_{r}^{2}}+1-2 \frac{m_{r}^{2}}{s}\right) \frac{m_{r}^{2}}{s-m_{r}^{2}}+\left(\frac{M_{W}^{2}}{m_{r}^{2}}+1+2 \frac{m_{r}^{2}}{s}\right) \frac{m_{r}^{2}}{t-m_{r}^{2}}\right]
\end{aligned}
$$

In the limit of large $s$, the leading order behavior of this amplitude is $s / \Lambda^{2}$, irrespective of the radion mass. This suggests that for sufficiently large $s$, the radion contribution is not perturbatively calculable and thus may violate unitarity. Following the analysis discussed in the previous section, the zeroth partial wave is easily obtained:

$$
\begin{aligned}
a_{0}=-\frac{g^{2}}{16 \pi} \gamma^{2}[ & \frac{s}{8 M_{W}^{2}}-\frac{3}{2}+\frac{m_{r}^{2}}{2 M_{W}^{2}}+\left(\frac{M_{W}^{2}}{m_{r}^{2}}+1+\frac{m_{r}^{2}}{4 M_{W}^{2}}+2 \frac{m_{r}^{2}}{s}\right) \frac{m_{r}^{2}}{s-m_{r}^{2}} \\
& \left.-\left(\frac{M_{W}^{2}}{m_{r}^{2}}+1+\frac{m_{r}^{2}}{4 M_{W}^{2}}-2 \frac{m_{r}^{2}}{s}\right) \frac{m_{r}^{2}}{s} \ln \left(1+\frac{s}{m_{r}^{2}}\right)\right] .
\end{aligned}
$$

The leading order term for $s \gg M_{W}^{2}, m_{r}^{2}$ can be rewritten compactly as

$$
\left.a_{0}\right|_{\text {leading }}=-\frac{1}{192 \pi} \frac{s}{\Lambda^{2}} .
$$

Thus, the radion mass does not regularize the bad high energy behavior of the partial wave amplitude because there are no particular symmetry relations between the radion and the Goldstone bosons. However, we have already argued that our 4-d effective theory is valid for energies only up to about the cutoff scale $\Lambda$. Under the condition $s, m_{r}^{2}<\Lambda^{2}$, the radion contributions will not saturate unitarity and thus no significant bounds can be obtained. 


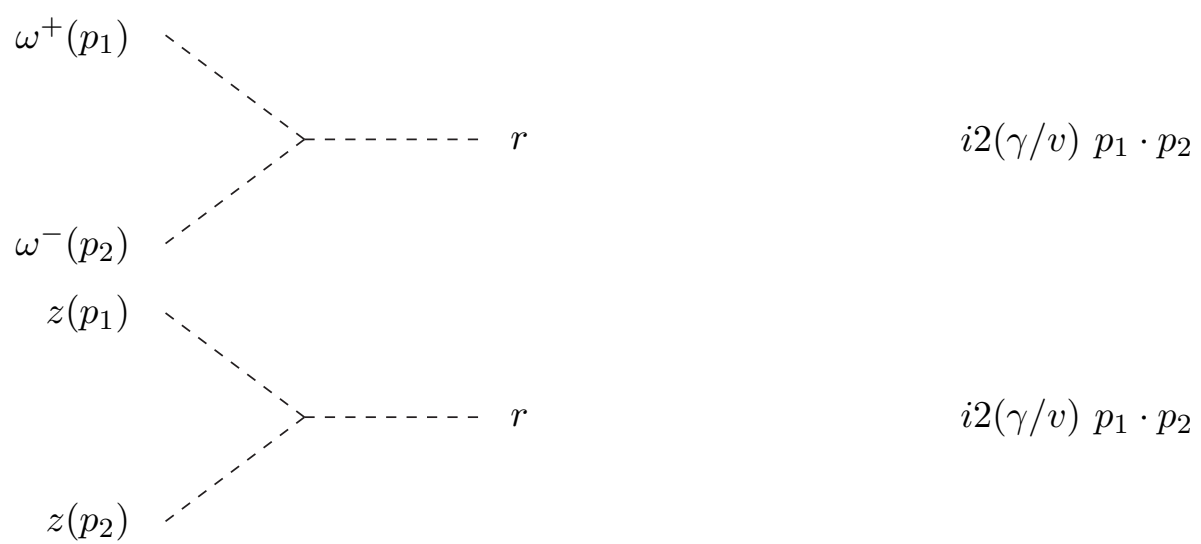

Figure 3: Feynman rules for the Goldstone bosons $\omega^{ \pm}$and $z$ with all momenta incoming.

\subsection{Radion interactions with electroweak Goldstone bosons}

The above analysis utilized the the gauge boson - radion Feynman rules while attaching longitudinally polarized $W$ s to the external lines. It is instructive to see the same calculation done more directly by simply considering the couplings of the radion to the Goldstone bosons. This can be done by starting with the fundamental couplings of the radion in Eq. (13), but writing out only the scalar kinetic and potential terms. This is equivalent to turning off the electroweak gauge couplings, $g=g^{\prime} \rightarrow 0$. In fact, only the kinetic terms are relevant

$$
S=\int d^{4} x \partial_{\mu} H^{\dagger} \partial^{\mu} H
$$

where $H=\left(-i \omega^{+}, \frac{1}{\sqrt{2}}(h+i z)\right)$ and $\omega^{ \pm}, z$ are the charged and neutral Goldstone bosons respectively. This results in the following interactions between the Goldstone bosons and the radion at $\mathcal{O}(r)$,

$$
\mathcal{L}=-\frac{2 \gamma}{v} r \partial_{\mu} w^{+} \partial^{\mu} w^{-}-\frac{\gamma}{v} r \partial_{\mu} z \partial^{\mu} z
$$

leading to the Feynman rules shown in Fig. 3. Using these Feynman rules we obtain the same leading order amplitude given by the terms in the first line of Eq. (15).

At this point, we should comment on this equivalence. The physical Higgs is part of the full Higgs doublet of the SM, and so its interactions are enforced by the electroweak symmetry. It is not immediately obvious that the Goldstone boson approximation automatically applies for radion interactions, since the latter are dimension five operators added to SM Lagrangian. In fact, naively one might add a curvature-Higgs mixing term to the scalar Lagrangian that is arranged to cancel the kinetic terms in the energy momentum tensor. This addition "improves" the energy momentum tensor by rendering it classically conformally invariant. However, as we will see in the next Section, the addition of curvature-Higgs mixing can be consistently treated in either prescription. Hence, the Goldstone boson approximation does indeed apply for the case with the radion, including when mixed with the Higgs. 
Since the radion contributions to the gauge boson scattering amplitude are suppressed by $\gamma^{2} \propto 1 / \Lambda^{2}$, there is no region where a large contribution is expected in the 4 -d effective theory, despite the $\mathcal{O}\left(s / M_{W}^{2}\right)$ divergence. This same result can be trivially applied to all other gauge boson scattering amplitudes involving the exchange of an (internal) radion. Fundamentally this result is straightforward to see from the radion interactions - the radion contribution is always suppressed by $1 / \Lambda$ instead of $1 / v$. There is, however, one important difference between the Higgs and the radion interactions: At one-loop the radion couples to trace anomaly gauge interaction [11, 12]. Here, the strength of the interaction is $\gamma b_{a} g_{a}^{2} / 16 \pi^{2}$, where $b_{a}$ is one-loop beta function coefficient for the $a$ coupling. This is generally much larger than the analogous one-loop coupling to the Higgs [at least for $a=\mathrm{SU}(3)$ ], scaling roughly with the number of heavy fermions running in the loop. Nevertheless, this is still suppressed relative to the size of tree-level couplings and thus does not give rise to any new unitarity problems.

\section{Curvature-Higgs mixing}

The couplings of the radion that we have used to calculate gauge boson scattering were independent of the Higgs couplings. This need not be the case, however. In particular, on the TeV brane we ought to add all terms to the action that are not forbidden by symmetries. One important term is a curvature-Higgs mixing operator [11]

$$
S^{\text {mixing }}=\int d^{4} x \sqrt{-g_{\text {ind }}} \xi H^{\dagger} H \mathcal{R}^{(4)}\left(g_{\text {ind }}\right),
$$

where $\xi$ is the dimensionless coefficient of this operator, $g_{\text {ind }}$ is the induced metric on the TeV brane and $\mathcal{R}^{(4)}\left(g_{\text {ind }}\right)$ is the 4 -d Ricci scalar written explicitly as a function of the induced metric. In theories with a flat extra dimension, this term gives rise to a tiny $1 / M_{\mathrm{Pl}}$ suppressed mixing between the radion and the Higgs. In RS1, however, this mixing is suppressed by the inverse warped Planck scale $1 / \Lambda$. The curvature-Higgs mixing operator induces kinetic mixing between the radion and the Higgs [11, []

$$
S^{\text {mixing }}=\int d^{4} x\left[6 \xi \gamma h \square r+3 \xi \gamma^{2}(\partial r)^{2}\right] .
$$

This mixing can be diagonalized by appropriate field redefinitions and rotations, resulting in the following relations between the interaction and mass eigenstates [0]

$$
\begin{aligned}
h & \rightarrow A h_{m}+B r_{m} \\
-\gamma r & \rightarrow C h_{m}+D r_{m}
\end{aligned}
$$

where

$$
\begin{aligned}
& A=\cos \theta-\frac{6 \xi \gamma}{Z} \sin \theta \\
& B=\sin \theta+\frac{6 \xi \gamma}{Z} \cos \theta \\
& C=\frac{\sin \theta}{Z} \gamma \\
& D=-\frac{\cos \theta}{Z} \gamma
\end{aligned}
$$




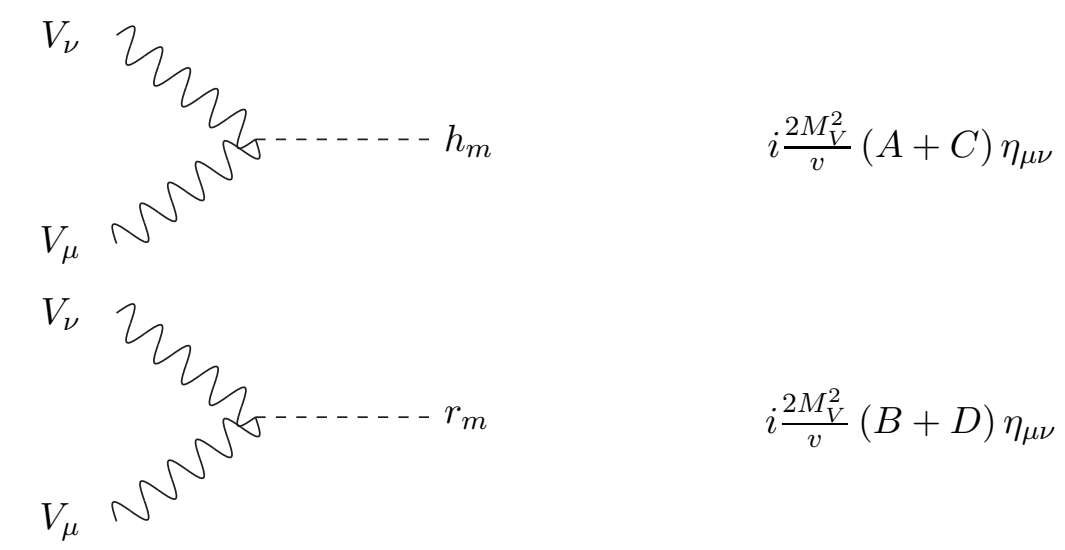

Figure 4: Feynman rules for three-point gauge boson couplings including curvature-Higgs mixing.

and

$$
\begin{aligned}
Z^{2} & =1+6 \xi \gamma^{2}(1-6 \xi) \\
\tan 2 \theta & =12 \xi \gamma Z \frac{m_{h}^{2}}{m_{r}^{2}-m_{h}^{2}\left(Z^{2}-36 \xi^{2} \gamma^{2}\right)} .
\end{aligned}
$$

The mass eigenstates are written as $h_{m}$ and $r_{m}$, although we should emphasize that these are mixed scalars with interactions that do not necessarily resemble the Higgs and radion interaction eigenstates. Nevertheless, in the limit that $\xi \rightarrow 0$, the mixing matrix becomes

$$
\left(\begin{array}{cc}
A & B \\
C & D
\end{array}\right) \stackrel{\xi \rightarrow 0}{\longrightarrow}\left(\begin{array}{cc}
1 & 0 \\
0 & -\gamma
\end{array}\right)
$$

and hence $h \rightarrow h_{m}$ and $r \rightarrow r_{m}$.

The Feynman rules for the mixed states can be easily read off from Eq. (14) after substituting Eq. (22). For completeness, we show them in Fig. 4 .

\subsection{Mass independent contributions to $a_{0}$}

Since the Higgs interaction is modified, the cancellation between the Higgs contribution shown in Fig. 1(d), (e) and the gauge contribution Fig. 1(a),(b),(c) is no longer complete. There are now several important contributions: the two mixed scalar (Higgs and radion) contributions, and the incompletely canceled gauge boson piece. The leading order, mass independent amplitude for the sum of these contributions is

$$
-i \mathcal{M}=-g^{2}\left((A+C)^{2}+(B+D)^{2}-1\right)\left[\frac{s}{4 M_{W}^{2}}+\frac{t}{4 M_{W}^{2}}+2 \frac{t}{s}\right] .
$$

Using Eqs. (23)-(25), the coefficient can be written as

$$
(A+C)^{2}+(B+D)^{2}=1+\left(\frac{1-6 \xi}{Z} \gamma\right)^{2}
$$




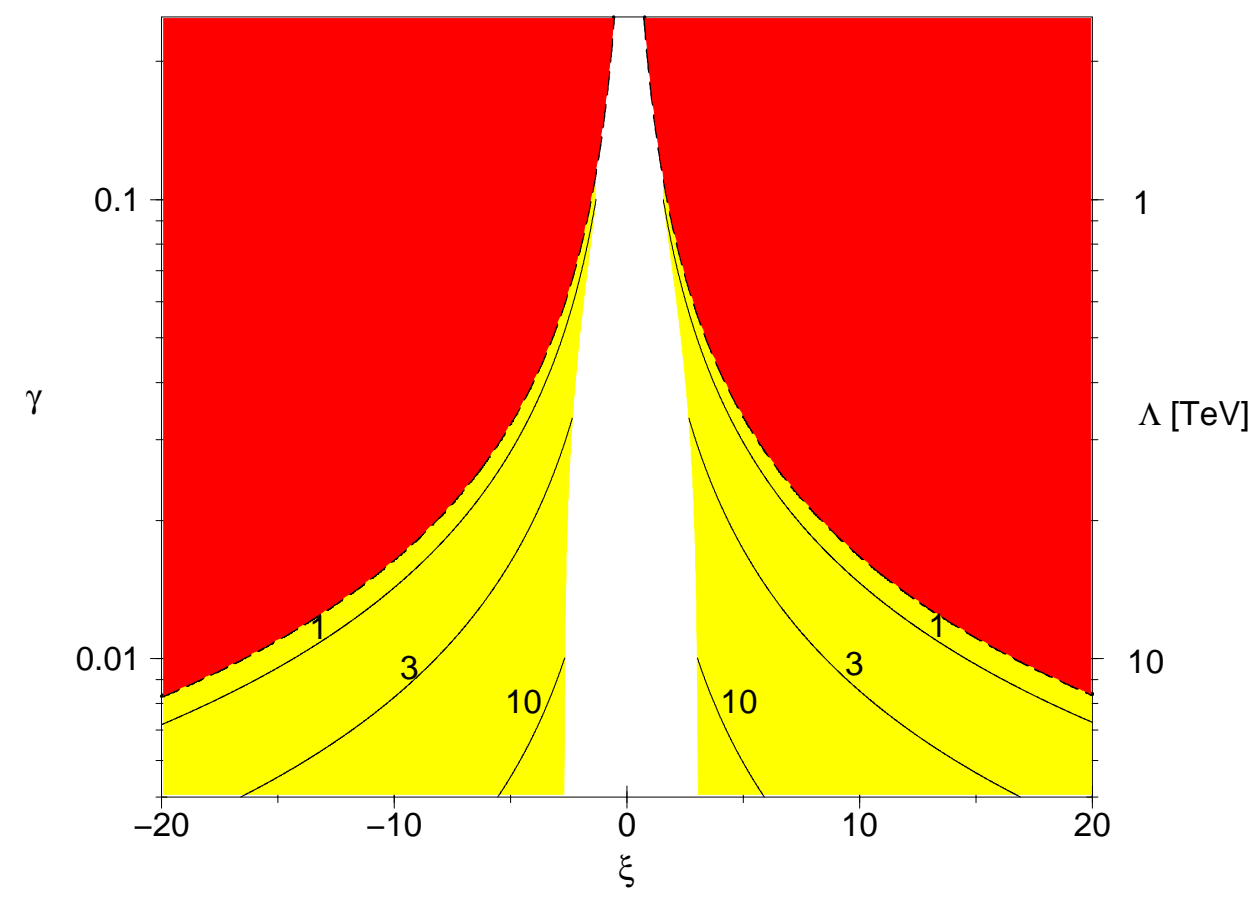

Figure 5: Bound on $\gamma=1 /(\sqrt{6} \Lambda)$ as a function of $\xi$. The dark shaded region (red) is excluded by requiring that kinetic terms for the Higgs and radion are positive (the crossover is shown by the dashed line). The light shaded region (yellow) is excluded by requiring that the gauge boson partial wave scattering amplitude does not exceed the perturbative unitarity bound prior to the $\mathrm{TeV}$ brane cutoff scale. The scattering energy $\sqrt{s}$ in $\mathrm{TeV}$ at which perturbative unitarity is violated is indicated by the contours in this region.

and so Eq. (27) reduces to

$$
-i \mathcal{M}=-g^{2}\left(\frac{1-6 \xi}{Z} \gamma\right)^{2}\left[\frac{s}{4 M_{W}^{2}}+\frac{t}{4 M_{W}^{2}}+2 \frac{t}{s}\right]
$$

Again, the same result can be obtained through the Goldstone boson approximation, as shown in the Appendix.

For small $\xi$ and $\gamma$, the coefficient of the $s / M_{W}^{2}$ term becomes

$$
(1-12 \xi) \gamma^{2}
$$

and so there is no significant enhancement of the scalar contribution to the gauge boson scattering amplitude. However, if $\xi$ is proportional to $1 / \gamma$, then the coefficient may not be suppressed by $\gamma^{2}$. For instance, suppose $\xi=-\epsilon / 6 \gamma$ with $\epsilon \lesssim 1$, then the coefficient becomes

$$
\frac{\epsilon^{2}}{1-\epsilon^{2}+\epsilon \gamma}
$$


which is $\mathcal{O}(1)$ and not suppressed by $\gamma^{2}$. Hence, for a large curvature-Higgs mixing coefficient there can be large contributions to the gauge boson scattering amplitude. The partial wave amplitude for the mass independent ("mi") terms is

$$
a_{0}^{\mathrm{mi}}=-\frac{g^{2}}{16 \pi}\left(\frac{1-6 \xi}{Z} \gamma\right)^{2}\left[\frac{s}{8 M_{W}^{2}}-\frac{3}{2}\right] .
$$

Requiring that this contribution to the partial wave amplitude does not exceed the perturbative unitarity bound implies an upper bound on the gauge boson scattering energy, for moderate or large $\xi$. We show the bound as a function of $\xi$ in Fig. 5. This is one of the central results of this paper. If we require that our 4-d effective theory is perturbative up to the TeV brane cutoff scale, meaning

$$
a_{0}(s)<\frac{1}{2} \quad \text { for all } s \text { up to } \Lambda^{2},
$$

then the light shaded region (yellow) is excluded. We find that $|\xi|$ must be less than about 2.7, for perturbative unitarity to be respected in our 4-d effective theory independent of the cutoff scale. As expected, for small $|\xi|$, the mass independent contribution never exceeds the perturbative unitarity bound, which is consistent with the discussion in Sec. 3.1. For $|\xi| \gtrsim 2.7$, we can identify the gauge boson scattering energy at which perturbation theory breaks down, which is shown by the contours. The contours halt at small $|\xi|$ when $\sqrt{s}=\Lambda$. The dark shaded region is excluded by requiring that the kinetic terms for the radion and the Higgs are positive definite, and so $Z^{2}>0$ in Eq. (24) [7]. No significant bound is obtained for the region $\Lambda \lesssim 1$ $\mathrm{TeV}(\gamma \gtrsim 0.1)$, since at best the leading order term is $\mathcal{O}\left(1 \mathrm{TeV}^{2} / M_{W}^{2}\right)$, and we already know that the $\mathcal{O}\left(m_{h}^{2} / M_{W}^{2}\right)$ term with $m_{h} \sim 1 \mathrm{TeV}$ is marginally allowed in the SM.

\subsection{Mass dependent contributions to $a_{0}$}

Up to now we have considered the mass independent terms to gauge boson scattering. The full result, including mass effects, is

$$
\begin{aligned}
a_{0}^{\text {total }}=a_{0}^{\mathrm{mi}}-(B+D)^{2} & {\left[\frac{m_{r_{m}}^{2}}{2 M_{W}^{2}}\left(\frac{M_{W}^{2}}{m_{r_{m}}^{2}}+1+\frac{m_{r_{m}}^{2}}{4 M_{W}^{2}}+2 \frac{m_{r_{m}}^{2}}{s}\right) \frac{m_{r_{m}}^{2}}{s-m_{r_{m}}^{2}}\right.} \\
& \left.-\left(\frac{M_{W}^{2}}{m_{r_{m}}^{2}}+1+\frac{m_{r_{m}}^{2}}{4 M_{W}^{2}}-2 \frac{m_{r_{m}}^{2}}{s}\right) \frac{m_{r_{m}}^{2}}{s} \ln \left(1+\frac{s}{m_{r_{m}}^{2}}\right)\right] \\
-(A+C)^{2}[ & \frac{m_{h_{m}}^{2}}{2 M_{W}^{2}}\left(\frac{M_{W}^{2}}{m_{h_{m}}^{2}}+1+\frac{m_{h_{m}}^{2}}{4 M_{W}^{2}}+2 \frac{m_{h_{m}}^{2}}{s}\right) \frac{m_{h_{m}}^{2}}{s-m_{h_{m}}^{2}} \\
& \left.-\left(\frac{M_{W}^{2}}{m_{h_{m}}^{2}}+1+\frac{m_{h_{m}}^{2}}{4 M_{W}^{2}}-2 \frac{m_{h_{m}}^{2}}{s}\right) \frac{m_{h_{m}}^{2}}{s} \ln \left(1+\frac{s}{m_{h_{m}}^{2}}\right)\right]
\end{aligned}
$$

where $m_{h_{m}}, m_{r_{m}}$ are the physical masses of the mixed Higgs/radion scalars. Notice that the scalar mixing coefficients are positive definite, and in fact from Eq. (28) it is obvious that $(A+C)^{2}>\frac{1}{2}$ 

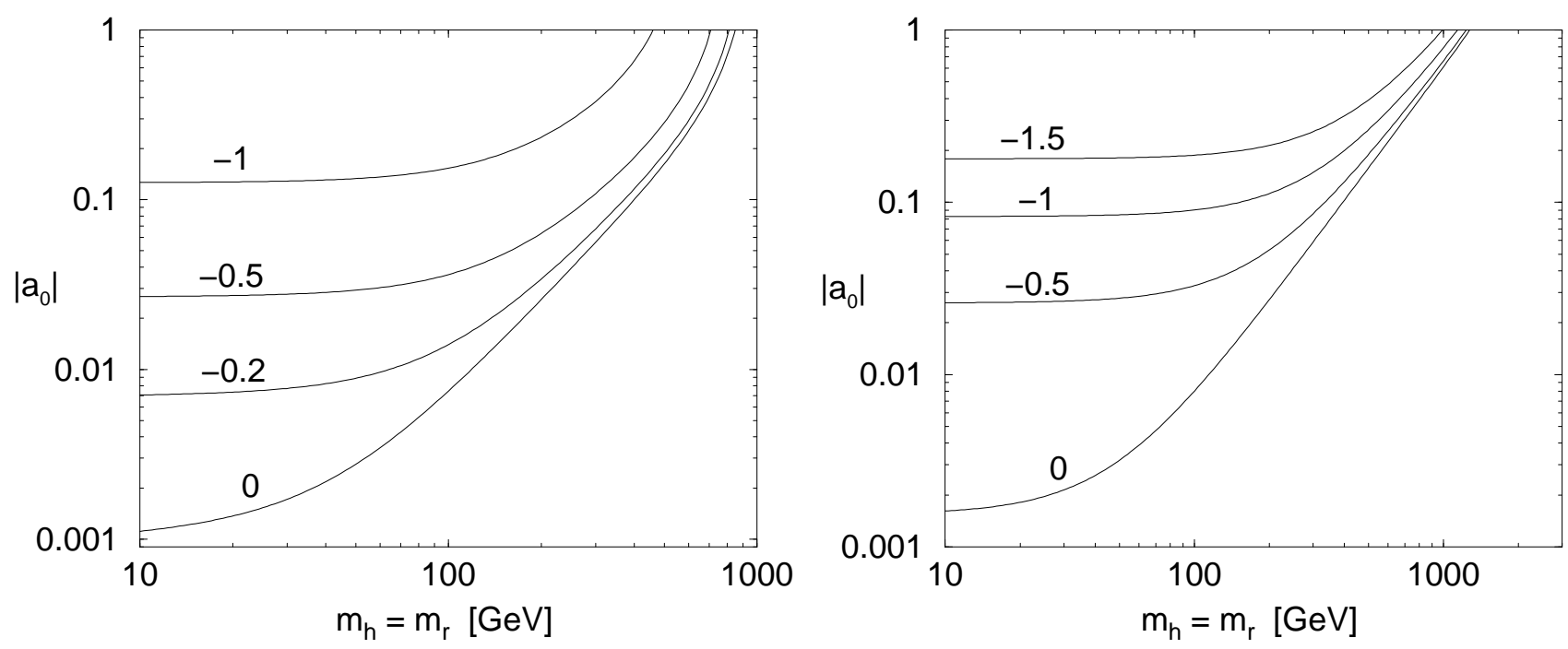

Figure 6: The contribution to the $j=0$ partial wave amplitude $a_{0}$ from all tree-level diagrams, including the mass effects of Higgs and the radion. The $x$-axis corresponds to the interaction eigenstate masses of the Higgs and radion (taken to be equal for illustration). The scattering amplitude was evaluated at the maximal energy $\sqrt{s}=\Lambda=(1,3) \mathrm{TeV}$ for the graph on the (left,right). Contours of various values of the curvature-Higgs mixing parameter $\xi$ are shown.

or $(B+D)^{2}>\frac{1}{2}$, and so there are always significant contributions to the partial wave amplitude from these mass terms. We illustrate this in Fig. 6 in two cases with $\sqrt{s}=\Lambda=1,3 \mathrm{TeV}$ by varying the masses and the curvature-Higgs mixing $\xi$, while fixing the interaction eigenstate masses to be equal. One has to take care in doing the calculation, since now there are poles in $1 /\left(s-m^{2}\right)$ for $m=m_{h_{m}}$ and $m=m_{r_{m}}$. With maximal $s=\Lambda^{2}$, the effects of these poles show up only when the mass is $\mathcal{O}(\Lambda)$, which is already in the region in which we cannot reliably calculate. The result is that the "allowed" region shown in Fig. 5 is further reduced by including the finite mass effects of radion and the Higgs.

The above results were obtained by assuming the masses of the two scalars are (approximately) equal. Even when the masses are widely separated, we find that the unitarity bound is reduced. We illustrate this in Fig. 目 where we show contours of $\left|a_{0}\right|=0.5$ as a function of the Higgs and radion masses, for the choice $\sqrt{s}=\Lambda=5 \mathrm{TeV}$ and various values of $\xi$. The interaction eigenstate masses are shown on the axes, although they are nearly the same as the the physical masses throughout the plot. Thus, requiring perturbative unitarity is not violated places upper bounds on the masses of the mixed scalars (radion and Higgs), in regions of $(\gamma, \xi)$ parameter space allowed by Fig. 5 .

The contributions to the partial wave amplitude above the Higgs and radion mass poles always constructively interfere, and so the presence of a radion universally increases the size of the partial wave amplitude. Below the mass of the Higgs and the radion $\sqrt{s} \ll m_{h}, m_{r}$, the partial wave amplitude is given by Eq. (10) with no dependence on the masses of the scalars, as the 


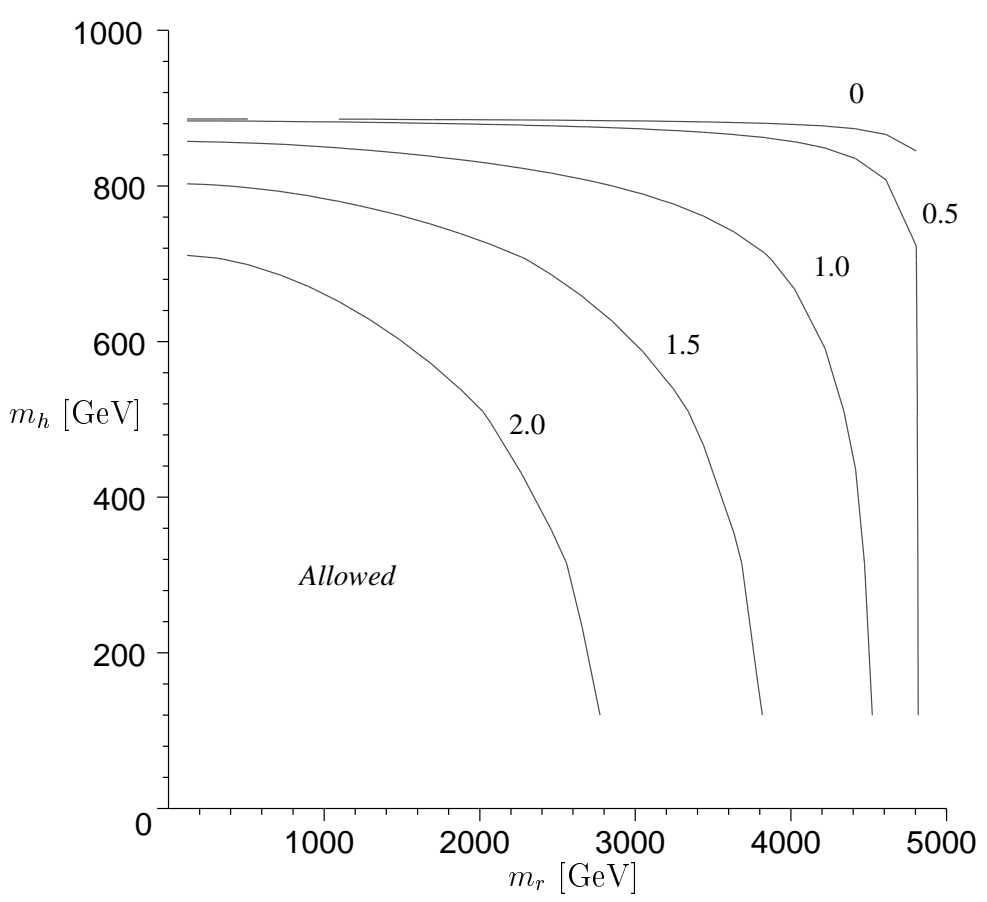

Figure 7: Constraints on the radion and Higgs masses from the perturbative unitarity bound. For this graph, we set $\sqrt{s}=\Lambda=5 \mathrm{TeV}$. Contours of various values of $\xi$ are shown with $a_{0}=0.5$. The allowed region lies below and to the left of the contours.

decoupling theorem requires. The remaining region, namely $m_{h}<\sqrt{s}<m_{r}$ (or $m_{r}<\sqrt{s}<m_{h}$ ) has the interesting behavior that the contribution from the Higgs and the radion have opposite sign due to the differing sign of the pole $m^{2} /\left(s-m^{2}\right)$. While this does not give rise to any new unitarity problems nor does it relax the above constraints, it is an interesting distinction as compared with the high energy behavior of the partial wave amplitude of the Higgs. In Fig. B we illustrate the differing behavior of the partial wave amplitude as a function of scattering energy. Notice that for scattering energies lower than the radion mass, the partial wave amplitude drops rapidly. This should be compared against the SM (or the "SM + radion" no mixing case) which asymptotes to a constant at large energy.

\section{Conclusions}

We have calculated the tree-level contribution to gauge boson scattering including the radion in the 4-d effective theory, valid below the cutoff scale $\Lambda=e^{-k L} M_{\mathrm{Pl}}$, obtained from the RandallSundrum solution to the hierarchy problem. In general, the radion provides a new contribution that is linearly divergent in $s$, going as $s / \Lambda^{2}$. This was calculated using longitudinally polarized gauge bosons with the three-point $W_{\mu}^{+} W_{\nu}^{-} r$ couplings, and independently using the eaten Goldstone bosons with their $\omega^{+} \omega^{-} r$ couplings. However, since our effective theory is only valid up to 


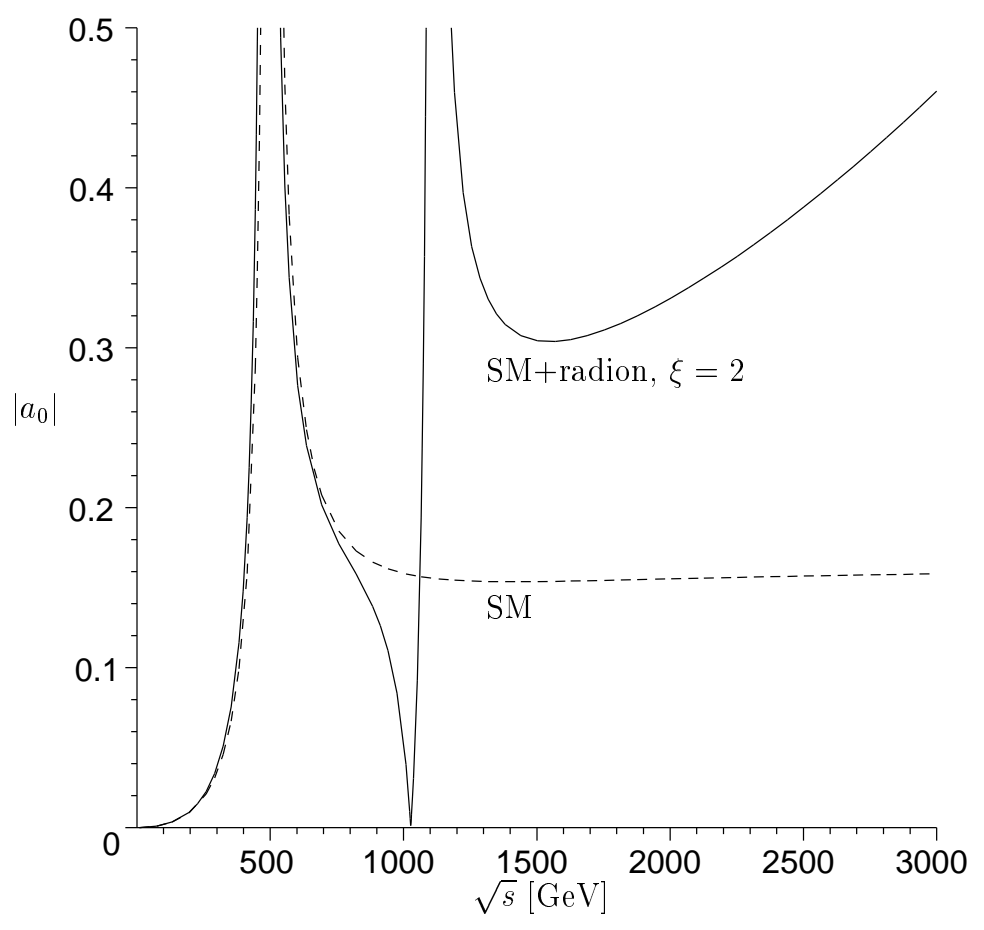

Figure 8: The partial wave amplitude as a function of $\sqrt{s}$. The dashed line is the SM for $m_{h}=500$ $\mathrm{GeV}$ (width effects were not taken into account). The solid line shows the behavior for the same Higgs mass but with a radion of mass $\sim 2 m_{h}$ with curvature-Higgs mixing in the spectrum. Notice that destructive interference occurs between $m_{h}<\sqrt{s}<m_{r}$, due to the differing sign of the (leading) pole contributions, which contrasts with the asymptotically flat behavior of the partial wave amplitude in the SM.

of order $\Lambda$, no significant bounds can be obtained when the Higgs and radion are mass eigenstates with no mixing. This linear contribution is exactly analogous to the Higgs, but unlike the Higgs there is no gauge cancellation between this term and ordinary gauge boson exchange.

Including curvature-Higgs mixing into the 4-d effective theory dramatically affects the gauge boson scattering cross section. In particular, we found that the mixing coefficient $|\xi|$ must be less than 3 so that perturbative unitarity is not violated prior to reaching the TeV brane cutoff scale $\Lambda$, independent of the mass of the radion and Higgs as seen in Fig. 5. It is interesting to remark that with the curvature-Higgs mixing coefficient $\xi \sim-1 /(6 \gamma)$, electroweak precision constraints can be satisfied with $m_{h}, m_{r} \gg M_{W}$ []]. We find, however, that this large mixing implies the theory must also become non-perturbative at a scale significantly below the TeV brane cutoff scale. We also calculated the partial wave amplitude including the radion and Higgs masses, and we found that the allowed region of $(\gamma, \xi)$ satisfying perturbative unitarity is further reduced. Mass bounds on the Higgs boson and the radion can be inferred for large $\xi$, as shown in Fig. 7 . Via our explicit calculations, we verified the validity of the Goldstone boson equivalence theorem with the existence of the curvature-Higgs mixing. 


\section{Acknowledgments}

G.D.K. thanks the theoretical physics group at LBL where part of this work was completed. This work was supported in part by the U.S. Department of Energy under grant number DEFG02-95ER40896, and in part by the Wisconsin Alumni Research Foundation.

\section{Appendix: Goldstone boson calculation including mixing}

Given the action

$$
S=\int d^{4} x \sqrt{-g}\left(g^{\mu \nu} D_{\mu} H^{\dagger} D_{\nu} H+\xi H^{\dagger} H \mathcal{R}^{(4)}\right)
$$

with the Higgs doublet $H=\left[-i \omega^{+}, \frac{1}{\sqrt{2}}(h+i z)\right]$ expressed in terms of the Goldstone bosons $\omega^{ \pm}$ and $z$. The induced metric expressed explicitly in terms of the radion field is [7]

$$
g_{\mu \nu}=e^{-2 k L-2(\gamma / v) r} \eta_{\mu \nu} .
$$

It is straightforward to carry out the rescalings of the fields $H \rightarrow e^{-k L} H$, etc., so that the curvature-Higgs mixing term $H^{\dagger} H \mathcal{R}^{(4)}$ can be expressed as $\left(\omega^{+} \omega^{-}+\frac{1}{2} z^{2}\right) \square r$ to $\mathcal{O}(r)$. Suitable manipulations of this term result in the Goldstone boson/radion interaction terms

$$
\mathcal{L}=-2 \frac{\gamma}{v}(1-6 \xi) r\left[\partial_{\mu} \omega^{+} \partial^{\mu} \omega^{-}+\frac{1}{2} \partial_{\mu} z \partial^{\mu} z\right]
$$

We have implicitly used the Landau gauge, setting $\square \omega^{ \pm}=\square z=0$.

Following the discussion in Sec. 4, we then rewrite the Lagrangian in terms of the physical eigenstates. To see that we obtain the same leading order mass-independent amplitude, we can make the simplification $m_{h}=m_{r}=0$ in Eq. (25), and then from Eq. (23) it is clear that $r=r_{m} / Z$. Using the interaction Lagrangian above, with the physical radion mass eigenstate $r_{m}$, the Goldstone boson partial wave amplitude is exactly the same as Eq. (30). It is also not hard to verify the leading order mass dependent terms given in Eq. (32) can be similarly obtained.

\section{References}

[1] L. Randall and R. Sundrum, Phys. Rev. Lett. 83, 3370 (1999) hep-ph/9905221.

[2] C. Csáki, M. Graesser, C. Kolda and J. Terning, Phys. Lett. B 462, 34 (1999) hep$\mathrm{ph} / 9906513$.

[3] W. D. Goldberger and M. B. Wise, Phys. Rev. Lett. 83, 4922 (1999) hep-ph/9907447.

[4] W. D. Goldberger and I. Z. Rothstein, Phys. Lett. B 491, 339 (2000) hep-th/0007065. 
[5] C. Charmousis, R. Gregory and V. A. Rubakov, Phys. Rev. D 62, 067505 (2000) hepth/9912160].

[6] O. DeWolfe, D. Z. Freedman, S. S. Gubser and A. Karch, Phys. Rev. D 62, 046008 (2000) hep-th/9909134.

[7] C. Csáki, M. L. Graesser and G. D. Kribs, Phys. Rev. D 63, 065002 (2001) hep-th/0008151].

[8] T. Tanaka and X. Montes, Nucl. Phys. B 582, 259 (2000) hep-th/0001092.

[9] C. Csáki, M. Graesser, L. Randall and J. Terning, Phys. Rev. D 62, 045015 (2000) hep$\mathrm{ph} / 9911406]$.

[10] W. D. Goldberger and M. B. Wise, Phys. Lett. B 475, 275 (2000) hep-ph/9911457.

[11] G. F. Giudice, R. Rattazzi and J. D. Wells, hep-ph/0002178.

[12] S. B. Bae, P. Ko, H. S. Lee and J. Lee, Phys. Lett. B 487, 299 (2000) hep-ph/0002224;

[13] U. Mahanta and S. Rakshit, Phys. Lett. B 480, 176 (2000) hep-ph/0002049; U. Mahanta and A. Datta, Phys. Lett. B 483, 196 (2000) hep-ph/0002183]; J. E. Kim, B. Kyae and J. D. Park, hep-ph/0007008; U. Mahanta, hep-ph/0008042; K. Cheung, Phys. Rev. D 63, 056007 (2001) hep-ph/0009232. S. C. Park, H. S. Song and J. Song, hep-ph/0009245; S. R. Choudhury, A. S. Cornell and G. C. Joshi, hep-ph/0012043.

[14] H. Davoudiasl, J. L. Hewett and T. G. Rizzo, Phys. Rev. Lett. 84, 2080 (2000) hepph/9909255; ibid., hep-ph/0006041.

[15] U. Mahanta, hep-ph/0004128.

[16] S. Bae, P. Ko, H. S. Lee and J. Lee, hep-ph/0103187.

[17] M. S. Chanowitz and M. K. Gaillard, Nucl. Phys. B 261, 379 (1985).

[18] B. W. Lee, C. Quigg and H. B. Thacker, Phys. Rev. D 16, 1519 (1977).

[19] J. M. Cornwall, D. N. Levin and G. Tiktopoulos, Phys. Rev. D 10, 1145 (1974); J. Bagger and C. Schmidt, Phys. Rev. D 41, 264 (1990); H. He, Y. Kuang and X. Li, Phys. Rev. Lett. 69, 2619 (1992).

[20] W. D. Goldberger and M. B. Wise, Phys. Rev. D 60, 107505 (1999) hep-ph/9907218;

H. Davoudiasl, J. L. Hewett and T. G. Rizzo, Phys. Lett. B 473, 43 (2000) hepph/9911262]; Y. Grossman and M. Neubert, Phys. Lett. B 474, 361 (2000) [hep-ph/9912408]; T. Gherghetta and A. Pomarol, Nucl. Phys. B 586, 141 (2000) hep-ph/0003129.

[21] H. Verlinde, Nucl. Phys. B 580, 264 (2000) hep-th/9906182; N. Arkani-Hamed, M. Porrati and L. Randall, hep-th/0012148; R. Rattazzi and A. Zaffaroni, hep-th/0012248. 